\title{
LA ACADEMIA EN ACCIÓN: APRENDIZAJE BASADO EN PROYECTOS EN ENTORNOS UNIVERSITARIOS
}

\section{ACADEMY IN ACTION: \\ LEARNING BASED ON PROJECTS IN UNIVERSITY ENVIRONIMENTS}

\author{
Alice Naranjo S. ${ }^{1}$ \\ Freddy Correa Lemus ${ }^{2}$ \\ Universidad Politécnica Salesiana, Guayaquil, \\ Ecuador,
}

\section{RESUMEN}

La academia en acción permite describir el proceso que en el aula facilita impregnar a los estudiantes del carisma salesiano e investigativo, del desarrollo de proyectos fundamentados en el uso de los conceptos académicos en la práctica, interactuando con la sociedad. Es la descripción de una experiencia real vivida en las 1 ORCID https://orcid.org/0000-0002-4386-2335 Docente de la carrera ingeniería de sistemas y computación, Universidad Politécnica Salesiana, Sede Guayaquil. Chambers \#227 y 5 de Junio, Guayaquil Telf. 042590630 Ext. $4550 \mathrm{~s} / \mathrm{n}$ CELULAR. berthanaranjo.ups.edu.ec $2 \quad$ Orcid. https://orcid.org/0000-0002-6176-1802 Estudiante Investigador, Carrera Ingeniería en Computación, Guayaquil, Ecuador, fcorreal@est.ups.edu.ec, Universidad Politécnica Salesiana aulas de clases de la Universidad Politécnica Salesiana Sede Guayaquil, Carrera Ingeniería en Sistemas, en la que los estudiantes transitan del ser individualista al ser social, activo y participativo, en la solución de problemas que afectan a la sociedad a través del conocimiento y las tecnologías. En este artículo se describe cómo aplicar una estrategia de aprendizaje basada en proyectos enmarcada en la tecnología y la acción.

\section{PALABRAS CLAVE}

Aprendizaje basado en proyectos, Tecnologías de Información y Comunicación, Sistema Preventivo, Educación Superior, Desarrollo de competencias. 


\section{ABSTRACT}

The academy in action allows to describe the process that in the classroom allows to impregnate the studentes of the salesian and investigative charisma, of the development of projects based on the use of academic concepts in practice, interacting with society. It is the description of a real experience lived in the classrooms of the Salesian Polytechnic University of Guayaquil, Systems Engineering Career, in which the students transit from the individualistic self to being social, active and participatory in solving problems that affect society through knowledge and technologies. This article describes how to apply a project-based learning strategy framed in technology and action.

\section{KEYWORDS}

Project-based learning, Information and Communication Technologies, Preventive System, Higher Education, Skills Development.

\section{INTRODUCCIÓN}

La academia en acción nos permite transitar por un camino en el que el aula se conecta con la investigación y la sociedad. Elementos de la educación universitaria en algunos casos desconectados, pero que en la actualidad toman vigencia en un modelo educativo y en un contexto en el que prima el ser humano y su esencia, en el que se pone de manifiesto la puesta del conocimiento para la ayuda de la cantidad de problemas que aquejan a la sociedad.

La sociedad ecuatoriana constituye el campo de acción de este trabajo, en especial, la ciudad de Guayaquil. En este contexto se trabajan elementos educacionales y las Tecnologías de Información y Comunicación para alcanzar el desarrollo de un profesional competente con alta calidad humana, que resolverá de forma eficiente los problemas de la sociedad, pues las Universidades deben constituirse en laboratorios que permitan experimentar para luego generar productos finales de una alta calidad, en base a la prueba, ensayo y error en el desarrollo de proyectos académicos que al final del proceso de enseñanza aprendizaje se transforman en proyectos integradores de aprendizaje significativo para los futuros profesionales.

En este artículo se describe el sistema preventivo de Don Bosco aplicado dentro del desarrollo de proyectos tecnológicos gestionados bajo la metodología de aprendizaje basada en proyectos (ABP).

\section{MODELO EDUCATIVO}

La Universidad Politécnica Salesiana (UPS) forma juventudes con el carisma salesiano, lo que significa que en la formación de un honrado ciudadano y un buen cristiano, tal como reza en su misión o razón de ser, está el sello característico de la salesianidad.

En nuestros días los jóvenes están expuestos a muchos peligros y tentaciones, a muchos riesgos y desenfrenos, que quizás en nuestro tiempo no existían con tanta permisividad y que ahora existe amparada en un marco legal que es permisivo en aspectos de drogadicción; así la violencia, el terrorismo, la pornografía infantil, los delitos sexuales, la violencia contra la mujer y los niños dan cuenta de la grave situación a la que se enfrenta esta juventud, la que podemos observar en noticiarios y la prensa escrita, pero existen universidades que se preocupan por los jóvenes y su formación en valores, en el desarrollo del ser humano consciente y transformador de la sociedad lo que permite dar oportunidades de mejora y superación a jóvenes en riesgo.

Es en estos espacios institucionales donde se desarrolla el proceso formativo $y$ transformador del ser humano, bajo el sistema preventivo de Don Bosco que usa métodos apropiados para calar y transformar a los jóvenes educándolos en la razón, religión y amor [1], 
principios que deben aplicar los docentes en el aula acorde a la filosofía del sistema preventivo de Don Bosco.

El término "razón" destaca, según la visión auténtica del humanismo cristiano, el valor de la persona, de la conciencia, de la naturaleza humana, de la cultura, del mundo del trabajo y del vivir social, o sea, el amplio cuadro de valores que es como el equipo que necesita el hombre en su vida familiar, civil y política [2].

Es significativo señalar que ya hace más de un siglo Don Bosco, patrono de la Universidad, daba mucha importancia a los aspectos humanos y a la condición histórica del individuo: a su libertad, a su preparación para la vida y para una profesión, a la asunción de las responsabilidades civiles en un clima de alegría y de generoso servicio al prójimo. Formulaba tales objetivos con palabras incisivas y sencillas, tales como "alegría", "estudio", "piedad", "'cordura", "trabajo", "humanidad". Su ideal de educación se caracteriza por la moderación y el realismo. En su propuesta pedagógica hay una unión bien lograda entre permanencia de lo esencial y contingencia de lo histórico, entre lo tradicional y lo nuevo.

El "amor" se traduce a dedicación del educador como persona totalmente entregada al bien de los educandos, estando con ellos, dispuesta a afrontar sacrificios y fatigas por cumplir su misión y amparados por la fe. Donde la labor docente no es trabajo, es una oportunidad de transformar al ser humano, ello requiere estar verdaderamente a disposición de los jóvenes, en profunda concordancia de sentimientos y capacidad de diálogo. Es típica y sumamente iluminadora su expresión: "Aquí, con vosotros, me encuentro a gusto; mi vida es precisamente estar con vosotros" [3]. Con acertada intuición dice de modo explícito: Lo importante es "no sólo querer a los jóvenes, sino que se den cuenta de que son amados" [4].
El educador debe ser auténtico, pues, participa en la vida de los jóvenes, se interesa por sus problemas, procura entender cómo ven ellos las cosas, toma parte en sus actividades deportivas y culturales, en sus conversaciones; como amigo maduro y responsable, ofrece caminos y metas de bien, está pronto a intervenir para esclarecer problemas, indicar criterios y corregir con prudencia y amable firmeza valoraciones y comportamientos censurables. En tal clima de "presencia pedagógica" el educador no es visto como "superior", sino como "padre, hermano y amigo" [5].

Ese ambiente de "familiaridad", es el que se vive en la Universidad y es la base del accionar docente, así es el trato entre educadores y jóvenes, en las instituciones salesianas. La larga experiencia de trabajo con los jóvenes de Don Bosco, le había llevado a la convicción de que sin familiaridad es imposible demostrar el amor, y que sin tal demostración no puede surgir la confianza, condición imprescindible para el buen resultado de la educación. El cuadro de objetivos, el programa y la orientación metodológicas sólo adquieren concreción y eficacia, si llevan el sello de un "espíritu de familia" transparente, o sea, si se vive en ambientes serenos, llenos de alegría y estimulantes.

La educación, que según el método de Don Bosco favorece una original promoción humana, exige al corazón y a la mente del educador atenciones precisas: adquirir sensibilidad pedagógica, adoptar una actitud simultáneamente paterna y materna, esforzarse por evaluar todo cuanto acontece en el crecimiento del individuo y del grupo, según un proyecto de formación que una, inteligencia y vigor, finalidad de la educación y voluntad de buscar los medios más idóneos para ella. Don Bosco solía decir que "la educación es cosa de corazón" [6,7].

Aplicando los pilares del sistema preventivo en el desarrollo de este artículo se consideran los siguientes elementos: 


\section{RESPETO DE LA DIGNIDAD DE CADA PERSONA}

Pues no considera distinción alguna, por razones de raza, color, sexo, lengua, religión, opinión política o de otro género, de origen nacional o social, de riqueza, de nacimiento o de otra condición.

\section{EDUCAR EN POSITIVO.-}

El arte de educar en positivo, es un aspecto que pone adelante al ser humano antes que todo saber o conocimiento, proponiendo el bien en vivencias adecuadas, en actividades formativas en el aula que permiten envolver y contagiar a los estudiantes en valores, permitiéndoles crecer como jóvenes de bien, donde el docente de forma oportuna detecta y corrige desviaciones preparándolo para una sólida formación de su carácter.

\section{DIFUNDIR UN CLIMA FAMILIAR.}

Donde el compartir en el aula es un espacio para estar juntos, vivir en un ambiente cálido, acogedor, hermoso y enriquecedor.

\section{ENSEÑAR TRABAJO COLABORATIVO Y COOPERATIVO}

Fortalece el trabajo en equipo que le permite al estudiante formarse en el diálogo y la colaboración, en donde en cada actividad descubre nuevas formas para hacer el bien a la sociedad a través de sus conocimientos, donde los derechos humanos están vigentes y se viven en el día a día en el compartir con compañeros con y sin discapacidad, en donde no existen fronteras ni barreras de ningún tipo.

\section{ENSEÑAR A VIVIR EN SOCIEDAD}

Se prepara al estudiante para la vida y para ser un honrado ciudadano y un buen cristiano, se lo forma como persona comprometida con la sociedad y convencido de que el mejor servicio que se puede brindar a los demás está dado con alegría y estando siempre alegres.

\section{PROPONER A LOS JÓVENES IDEALES DE VIDA QUE LOS TRASCIENDE.}

A través de trabajos en clase o trabajos con la sociedad los estudiantes viven y aprenden la importancia de trascender a otro nivel y comprenden de mejor manera conceptos como: la paz, la libertad, la justicia, la equidad, la diversidad, la solidaridad, la caridad, la comunión y participación, el respeto a la mujer, la cordialidad, el desarrollo de un mundo sin contaminación y la atención a las necesidades ecológicas, para vivir en un mundo mejor.

Con todos los antecedentes descritos y analizados los elementos que prevalecen en este modelo de sistema educativo surge "la academia en acción", proyecto mediante el cual transformamos al ser humano en un ser-acción.

Con una diversidad de iniciativas, actividades y oportunidades que se les brinda a los jóvenes al transitar por las aulas. El convivir en un aprendizaje basado en un sistema preventivo se convierte en una experiencia memorable, pero esto no es tarea de un solo docente, es parte de una filosofía que se difunde en el aula por todos los docentes en la UPS. Entre las diversas metodologías, estrategias y técnicas a usar se encuentra el ABP, propuesta que difiere mucho de lo que se trabaja en el aula tradicional. En este artículo describiremos la metodología de aprendizaje basado en proyectos, la cual permite evaluar los aprendizajes de forma gradual haciendo uso de técnicas específicas que son propios de este enfoque.

\section{APRENDIZAJE BASADO EN PROYECTOS}

Se considera que el ABP surgió en Estados Unidos a finales del siglo XIX, comúnmente es conocido como una estrategia de enseñanzaaprendizaje que se basa en "el hacer", el educador William H. Kilpatrick elaboró el concepto y lo 
publicó en su artículo denominado "The Proyect Method".

El Aprendizaje Basado en Proyectos ( $A B P \circ P B L$, Project-based learning) es un modelo de enseñanza fundamentado en la utilización de proyectos auténticos y realistas, basados en una cuestión, tarea o problema altamente motivador y envolvente, relacionados directamente al contexto de la profesión [8,9], en él participan los alumnos y se fomenta el desarrollo de las competencias en un enfoque colaborativo trabajando en equipos estructurados en busca de soluciones a los problemas de la sociedad [9]. Otros autores lo identifican como una metodología de aprendizaje, un ambiente de aprendizaje activo centrado en los/las estudiantes y una técnica didáctica que se enfoca en un producto o en un proceso que los/las estudiantes tienen que planificar, diseñar y desarrollar a través de un proyecto [10-12].

Este modelo de aprendizaje exige que el profesor sea un creador y un guía que estimule a los estudiantes a aprender dado que la realidad se acerca al estudiante por medio de la realización de un proyecto que le permite dar respuesta a un problema planteado y lograr así el desarrollo de competencias. Como dice Arboleda [13], en este horizonte de las demandas educativas del mercado no es posible poder formar actitudes para la vida. Lo que sí es viable es formar en valores y actitudes para el trabajo, en las competencias ciudadanas que precisa el mundo laboral: ciudadanos que entregan su saber y su ser al servicio utilitario. Si los conocimientos y potenciales ciudadanos formados desde la escuela en el marco de las competencias se usan bajo los presupuestos de la economía global (léase, voraz), es improbable que al unísono sirvan los fines nobles (utópicos) de una sociedad más equitativa y justa.

Para poder desarrollar el ABP bajo el enfoque propuesto en [13] es importante mantener altamente motivados y comprometidos a los estudiantes, el aplicar los conocimientos adquiridos para evidenciar el desarrollo de competencias, es lo que hace que el trabajo se vuelva cada vez más demandante y logre estimular la participación de todo el equipo de estudiantes que desarrolla el proyecto. En este aprendizaje se atraviesa e interactúa con diversas disciplinas y asignaturas con lo que se produce la integración de conocimientos adquiridos previamente que le dan un sentido a lo aprendido.

\section{METODOLOGÍA}

Se aplicó la metodología ABP para el desarrollo de proyectos tecnológicos en la asignatura de arquitectura de computadoras en la UPS Salesiana, Carrera de Ingeniería de Sistemas en el año 2018, aunque es necesario destacar que esta metodología se ha aplicado desde el 2014 hasta la actualidad, en el presente estudio se evidencia el trabajo desarrollado en un paralelo de 40 estudiantes.

Se aplicó un cuestionario para conocer si la metodología ABP se había usado anteriormente por parte de los estudiantes en alguna asignatura y con el enfoque en $\mathrm{ABP}$, finalmente luego de la experiencia, se realizó un cuestionario para evaluar los resultados de la aplicación del ABP.

En el desarrollo del trabajo se aplica la metodología $\mathrm{ABP}$ con adaptaciones para el contexto. Se describen los componentes importantes del proceso, los cuales para este caso son: docente, estudiantes y asociaciones, federaciones o fundaciones de la red RAITI (Red Académica de Apoyo e Investigación en Tecnologías inclusivas).

El docente con su rol de director de proyectos ofrece a los alumnos una guía, provee de los recursos necesarios y brinda el apoyo, a cada equipo acorde a sus necesidades y en función del proceso investigativo que los equipos de trabajo van desarrollando. 
El docente actúa como facilitador plantea los problemas a resolver y cada grupo de estudiantes organizado en equipos acorde a su dominio y por afinidad establece el problema que adopta, de él generan el proyecto estableciendo los objetivos.

Los alumnos por su parte colaboran y trabajan de forma cooperativa en el desarrollo del proyecto, existe una amplia comunicación con el docente, recopilan y analizan información, experimentan y hacen pruebas del prototipo requerido y se va informando en las reuniones de avance los resultados obtenidos, los estudiantes se autoevalúan pero el maestro también forma parte del proceso de evaluación. El Aprendizaje basado en Proyectos favorece que se utilice una amplia variedad de estrategias y mecanismos de evaluación.

La red RAITI integrada por asociaciones, federaciones y fundaciones de personas con discapacidad, son las demandantes de proyectos en base a las necesidades concretas de su organización.

Las etapas del ABP que docentes y estudiantes aplicaron en el desarrollo de proyectos en el aula son:

\section{FORMACIÓN DE EQUIPOS DE TRABAJO}

Los estudiantes forman grupos de forma diversa, pero son ellos los que deciden con quien agruparse. Se establecen los roles de cada integrante, sólo dos son posibles: líder y colaborador.

Como elemento clave para el trabajo en equipo se introduce el tema "Conociendo los valores", los cuales se aplicarán a lo largo del periodo académico, para fortalecer en ellos aspectos como perseverancia, responsabilidad y respeto. Posteriormente se describen los lineamientos del "trabajo en equipo", que implica la búsqueda de relaciones armoniosas en la sociedad con el respeto hacia lo que siente, piensa y dice el otro, se explican aspectos de liderazgo para empoderar al líder a alcanzar la meta del trabajo en equipo.

\section{PLANTEAMIENTO DE LA PREGUNTA INICIAL}

A los diversos equipos de trabajo se los enfrenta a una pregunta de estudio por parte del docente, así se debe brindar a través de sus conocimientos previos y de la asignatura Arquitectura de computadoras, más autonomía a las personas con discapacidad en la interacción con el computador. En el planteamiento se alude a diversas discapacidades.

\section{DEFINICION DE LA SOLUCIÓN Y ESTABLECIMIENTO DEL PROYECTO}

Del problema establecido se determina el proyecto a realizar con el fin de favorecer el uso de las TIC a las personas con discapacidad. Los estudiantes seleccionan la discapacidad y entidad de la red RAITI con la que desean interactuar en el desarrollo de su proyecto y definen el escenario a resolver y el prototipo a construir. Para construir el prototipo deben usar las TIC: hardware, software, microcontroladores, tarjetas arduino, componentes electrónicos, periféricos adaptados, entre otros componentes y se deben integrar los conocimientos adquiridos en la asignatura y los conocimientos previos de otras asignaturas, para poder alcanzar la meta.

Cada grupo trabaja en dar solución al problema identificado y en ese proceso van descubriéndose unos a otros y aprendiendo a convivir con el otro, a respetar al prójimo pese a sus diferencias, adquieren conocimientos y aplican sus aprendizajes.

\section{INVESTIGACIÓN Y DOCUMENTACION DE LA SOLUCIÓN}

En esta etapa se inicia el proceso de investigación para poder solventar la solución, así se van cumpliendo algunos descriptores y parámetros del conocimiento mínimo acorde a 
la planificación macro, messo y microcurricular que el perfil profesional de Ingeniería de Sistemas exigió hasta el 2018 y que en la actualidad se mantiene bajo el nombre de Ingeniería en Computación, pero además se trabaja el componente social, con ello se va un paso hacia adelante en la formación del ser humano, en la preparación del futuro profesional para la vida.

Ese transitar de lo teórico (academia) a lo práctico y lo aplicado en la vida real, le da un mayor simbolismo a lo que aprendemos, más aún si el beneficio es para personas con discapacidad (PCD) que lo requieren.

La forma y el estilo de aprendizaje bajo este enfoque es totalmente diferente al acostumbrado puesto que pasamos del paradigma del conocimiento al paradigma de la acción y el descubrimiento.

\section{EJECUCIÓN/PRODUCCIÓN}

La fase de "ejecución" implica que cada grupo inicie la creación del prototipo con todo lo investigado, con todo lo relevado en la organización. El prototipo es el elemento indispensable para la concreción de la alternativa de solución que cada grupo construye, pero para ello se interactúa con la sociedad, con los integrantes de la asociación, federación o fundación de personas con discapacidad quienes lo van retroalimentando en el cumplimiento de requisitos planteados para el desarrollo del prototipo.

Así poco a poco en el período académico los proyectos van tomando forma y el prototipo basado en TIC y en conocimientos impartidos en la Carrera de Ingeniería en Sistemas, hoy Computación surge como respuesta al problema planteado. Este proceso puede tomar entre 4 a 5 meses y en él se canalizaron todos los ajustes necesarios. Al final, la creatividad de cada uno de los jóvenes se ha concretado y se va respondiendo a las necesidades planteadas por las asociaciones y federaciones a través de cada uno de los proyectos aceptados.

\section{IMPLEMENTACIÓN}

En esta etapa se pone en funcionamiento el prototipo en la organización, se recibe la aceptación del producto y a manera de "presentación del informe final" se efectúa una exposición del trabajo realizado ante los demás compañeros del aula, con esto se contribuye a la fase de difusión, etapa que demanda el ABP y que contribuye a la socialización de conocimientos con los compañeros así como al cierre del proyecto.

\section{RESULTADOS}

Como resultados en la primera parte de la evaluación que comprende el proceso previo a la implementación de la metodología del ABP se pudo evidenciar a través de la encuesta en línea aplicada, que hasta el 2018 ninguno de los estudiantes había participado en un proceso de este tipo, ni había implementado un proyecto técnico usando esta metodología durante la academia en asignaturas previas, no había integrado asignaturas, ni implementado un proyecto en alguna entidad.

Luego de aplicada la metodología en la asignatura Arquitectura de Computadoras se evaluó el post proceso y se obtuvo que el aprendizaje basado en proyectos permitió a los estudiantes desarrollar el aprendizaje significativo que dio sentido a lo que hacían en la academia y que al final fue implementado en cada una de las organizaciones.

Entre los indicadores resultantes que más se destacaron en el proceso tenemos que el $100 \%$ de los equipos de trabajo creados desarrollaron sus prototipos, culminaron su proyecto y lo entregaron a las entidades de la red RAITI.

Los grupos se mantuvieron unidos hasta el final, ninguno desertó, alcanzando el objetivo y desarrollando las competencias establecidas en el proceso. 
Las entidades, asociaciones, fundaciones, federaciones de personas con discapacidad recibieron su prototipo funcional concluido e implementado en su organización.

En total, 10 prototipos fueron entregados a las 10 entidades de la red RAITI para su uso por alrededor de 500 beneficiarios.

Los prototipos o productos softwares desarrollados fueron aceptados por cada una de las entidades de la red RAITI que participaron en este proceso.

El proceso resultó exitoso porque los estudiantes alcanzaron la meta que era el desarrollo de prototipos vinculado a TIC adaptativas dentro de la asignatura Arquitectura de Computadoras y en dicho proceso aprendieron a trabajar en equipo, con compañeros así como con entidades usuarias, desarrollando competencias necesaria en la Carrera.

\section{CONCLUSIONES}

El ABP es una estrategia educativa que consigue involucrar al estudiante en su propio aprendizaje y ser el constructor del mismo, a la vez que permite que el docente acompañe todo el proceso activo hasta lograr obtener la solución a una necesidad de la sociedad.

Esta metodología es una forma de encontrar sentido a los contenidos y aprendizajes, a la transversalidad de la inclusión y discapacidad como a la interdisciplinariedad de las asignaturas, y se efectúa a través de una particular forma diferenciada, la atención a problemas que aquejan a la sociedad desde la academia.

Transformaciones y manifestaciones de cambio, que se observaron en el proceso fueron muchas, puesto que cada una de las etapas permitió que cada estudiante vaya creciendo en valores y en responsabilidades que le toca asumir para poder garantizar entre todos, la culminación y el cierre del proyecto, competencia importante en su vida profesional.

Atender a sectores desatendidos de la sociedad y concretamente a las personas con discapacidad, nos permite generar espacios en la academia, docencia e investigación para nuestros alumnos, al mismo tiempo que se desarrolla la solidaridad con el otro y el apoyo a favorecer su mejora personal, proceso en el que todos ganan, tanto el alumno como los integrantes de la institución beneficiaria del proyecto.

La verdadera finalidad de un educador que enseña Tecnología consiste en ayudar a los estudiantes a que contribuyan de manera significativa con sus comunidades en la solución de problemas reales, a reducir las inequidades y desigualdades en el campo social, educativo o laboral, en resumen a reducir la exclusión de las personas con discapacidad. El aprendizaje basado en proyectos brinda esa oportunidad de integrar, academia, investigación y sociedad.

\section{REFERENCIAS BIBLIOGRÁFICAS}

[1] II Sistema Preventivo, en "Regolamento per le case della Società di S. Francesco di Sales", Turín 1877, in Giovanni Bosco, Scritti pedagogici e spirituali (AA.VV.), LAS, Roma 1987, pág. 166.

[2] «Carta Encíclica Redemptor hominis,», (1979), vol. AS , n 71, pp. 282. 284-285.

[3] Canavese S. B. (1904), «Memorias biográficas de San Juan Bosco,» vol. IV, p. 654.

[4] "Carta de Roma,», (1884), Scritti pedagogici e spirituali (AA.VV.), p. 294.

[5] «Carta de Roma,» Scritti pedagogici e spirituali , p. 296.

[6] M. b. d. S. J. Bosco, (1935), vol. XVI, p. $447,1935$.

[7] Canaves S. B. (1907), «Memorias bi- 
ográficas de San Juan Bosco,» vol. VI, pp. 815816.

[8] Bender W., Aprendizagem Baseada em Projetos: educação diferenciada para o século XXI (en portugués), Porto Alegre: Penso, 2014.

[9] MARTÍ, J., Heydrich, M., Rojas, M., \& Hernández, A. (2010). Aprendizaje basado en proyectos. Revista Universidad EAFIT, 46(158), 11-21.

[10] Caballero, E. et al. (2014). El aprendizaje basado en proyectos y la autoeficacia de los/las profesores/as en la formulación de un plan de clase. Revista Alteridad, 9(1), 56-64.

[11] Landron, M. L., Montoro, M. A., \& Ruiz, M. J. C. (2018). El efecto del aprendizaje basado en proyectos en estudiantes con altas capacidades intelectuales de una segunda lengua. Revista de educación, (380), 210-236.

[12] Guedes, M., Lourenco, J., Filipe, A. Almeida, L., \& Moreira, M. C. (2007). Ensino e aprendizagem por projecto. Lisboa, Portugal: Centro Atlantico.

[13] Arboleda, J. C. (2016). Formación para la vida: de las competencias a la comprensión edificadora. Revista Boletín Redipe, Vol. 4, №. 12, 2015, págs. 20-25. 